\title{
Order-disorder transition in the Cd-Ca cubic approximant
}

M. Widom and M. Mihalkovič ${ }^{1}$

Department of Physics, Carnegie Mellon University, Pittsburgh, PA 15213

${ }^{1}$ also at: Institute of Physics, Slovak Academy of Sciences, 84228 Bratislava, Slovakia

\begin{abstract}
Recent experiments discovered an order-disorder transition occuring at low temperatures in large unit 1/1 cell cubic approximants of the stable Cd-based binary alloy quasicrystals. The transition is related to correlations among orientational degrees of freedom whose separations are around $12 \AA$. We analyze the interactions between the degrees of freedom using ab-initio calculations for $\mathrm{Cd}-\mathrm{Ca}$ alloys and derive an equivalent antiferromagnetic Ising model which shows a similar phase transition. However, the calculated transition temperature is higher than observed experimentally, indicating that the actual structure and its order-disorder transition are more complex than originally proposed. A side-benefit of our study is the discovery of a canonical-cell decoration model for the Cd-Ca icosahedral phase.
\end{abstract}

\section{INTRODUCTION}

Stable icosahedral quasicrystals occur in the compounds $\mathrm{Cd}_{5.7} \mathrm{Yb}$ and $\mathrm{Cd}_{5.7} \mathrm{Ca}[1,2,3]$. In each case the phase diagram of the binary alloy contains a $1 / 1$ cubic approximant at a close-by composition $[4,5]$. The $\mathrm{Cd}_{6} \mathrm{Yb}$ structure [6] can be represented in a conventional simple cubic unit cell with a lattice parameter of $a=15.7 \AA$. The Pearson symbol for $\mathrm{Cd}_{6} \mathrm{Yb}$ is cI176 indicating that it is cubic, body-centered, with 176 atomic positions per simple cubic unit cell. Since 176 is not a multiple of the basic 7-atom stoichiometric unit (6 $\mathrm{Cd}$ and $1 \mathrm{Yb}$ ), there must be partial occupancy. In fact, the unit cell contains $144 \mathrm{Cd}$ atoms, $24 \mathrm{Yb}$ atoms and 8 vacancies. Sets of 4 vacancies alternate with sets of $4 \mathrm{Cd}$ atoms among vertices of a cube to form tetrahedra located at the center of a dodecahedral cluster (see Fig. 1). There are two such clusters per simple cubic cell, for a total of 8 vacancies.
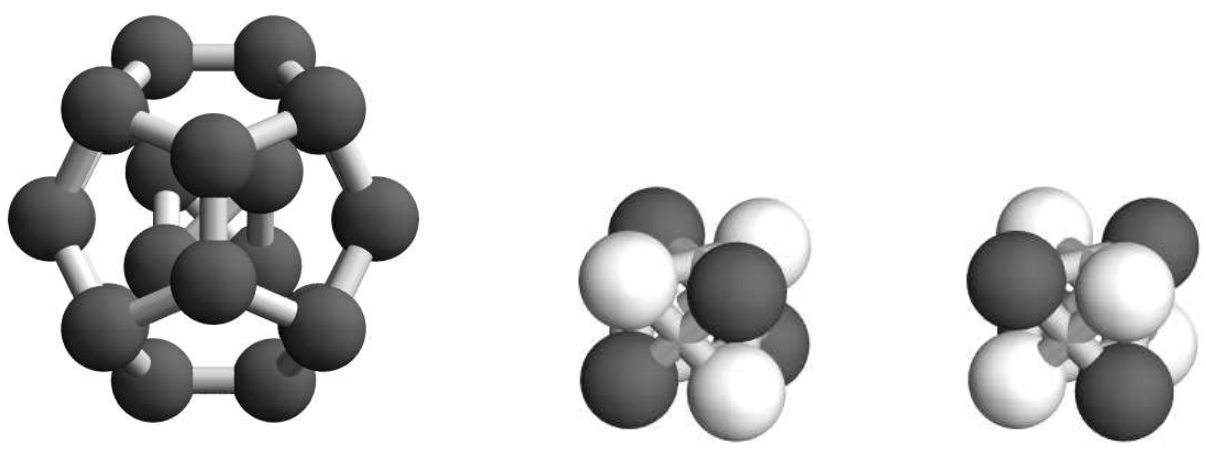

Figure 1: (Left) Innermost $\mathrm{Cd}_{20}$ dodecahedral shell centered by $\mathrm{Cd}_{4}$ tetrahedron, viewed along cubic [100] axis. (Right) Two alternate tetrahedral orientations (dark=Cd, light=vacancy) according to cI176 structure [6]. 
The proper structure type of $\mathrm{Cd}_{6} \mathrm{Ca}$ has recently been debated. Initial reports [7] assigned it to prototype $\mathrm{Cd}_{6} \mathrm{Y}$ with Pearson symbol cI184. This structure is equivalent to the cI176 structure of $\mathrm{Cd}_{6} \mathrm{Yb}$ except for the central $\mathrm{Cd}_{4}$ tetrahedra. In cI184 these $\mathrm{Cd}$ atoms occupy 4 out of 12 vertices of a cuboctahedron (resulting in 6 possible orientatons) instead of 4 out of 8 vertices of a cube (resulting in 2 possible orientations). After the discovery of stable $\mathrm{Cd}-\mathrm{Ca}$ quasicrystals some researchers $[8,9,10]$ suggested the structure type of $\mathrm{Cd}_{6} \mathrm{Ca}$ might actually be $\mathrm{Cd}_{6}$ Yb.cI176. The full story has yet to be resolved. Indeed a recent study of $M \mathrm{Cd}_{6}$ for a variety of metal atoms $M$ finds evidence for yet additional site types [11].

In order for the overall structure to be truly body-centered, it is necessary that either the tetrahedra at each center be identically aligned or else that all body centers be randomly oriented. In each case a body-center translation leaves the structure invariant. However, recent experiments indicate order-disorder transitions in which a supercell ordering develops at low temperatures, breaking the body-centered cubic symmetry $[10,12,13]$.

Estimated transition entropies close to $k_{B} \ln 2$ suggest that the cluster center tetrahedra have two equivalent orientations. Both should occur randomly at high temperatures, and freeze into some definite ordered pattern at low temperatures. Such a local two-state system can be modeled using the Ising model, with the two spin states representing the two cluster orientations. Because the cI176 structure of $\mathrm{Cd}_{6} \mathrm{Yb}$ possesses precisely two tetrahedron orientations, we began our initial study of the order-disorder transition using this structure.

\section{TOTAL ENERGY CALCULATIONS}

We carry out ab-initio calculations using the plane-wave program VASP [14, 15] which yields reasonably accurate total energies. This approach uses ultrasoft pseudopotentials [16] or PAW potentials [17] to represent the effective interaction of valence electrons with ionic cores, and solves the many-body quantum mechanical band structure of these electrons using electronic density functional theory. We choose to model $\mathrm{Cd}-\mathrm{Ca}$ rather than $\mathrm{Cd}-\mathrm{Yb}$ because the alkali earth element $\mathrm{Ca}$ is easier to treat from first principles than the rare earth element $\mathrm{Yb}$.

First, we use VASP to reproduce the sequence of low-temperature stable phases in the established $\mathrm{Ca}-\mathrm{Cd}$ binary phase diagram [5]. To do this we calculate the cohesive energy for each known structure, and several hypothetical ones. Each structure is fully relaxed in both unit cell parameters and atomic coordinates. All energies are converged to an accuracy of $1 \mathrm{meV}$ /atom or better by increasing the k-point mesh density. These calculations use PAW potentials in the generalized gradient approximation and a constant plane-wave energy cutoff of $274 \mathrm{eV}$.

Subtracting each cohesive energy from the tie-line joining the pure elements in their ground states yields enthalpies of formation (at $\mathrm{T}=0 \mathrm{~K}$ ). Enthalpies of all known and many hypothetical Ca-Cd structures are plotted in Fig. 2. We label each structure with its name followed by its Pearson symbol.

Agreement between our calculation and the established phase diagram requires that all known low temperature structures lie on the convex hull of enthalpy versus composition. Additionally, all hypothetical structures must lie above the convex hull, as must all known high temperature, high pressure and metastable phases. Agreement is perfect, except for one seeming difficulty with $\mathrm{CaCd}_{2}$ which we now address.

The low and high temperature $\mathrm{CaCd} 2$ phases are reversed in energy relative to the experimental report [5]. However, the transition between the hP12 and oI12 variants has 


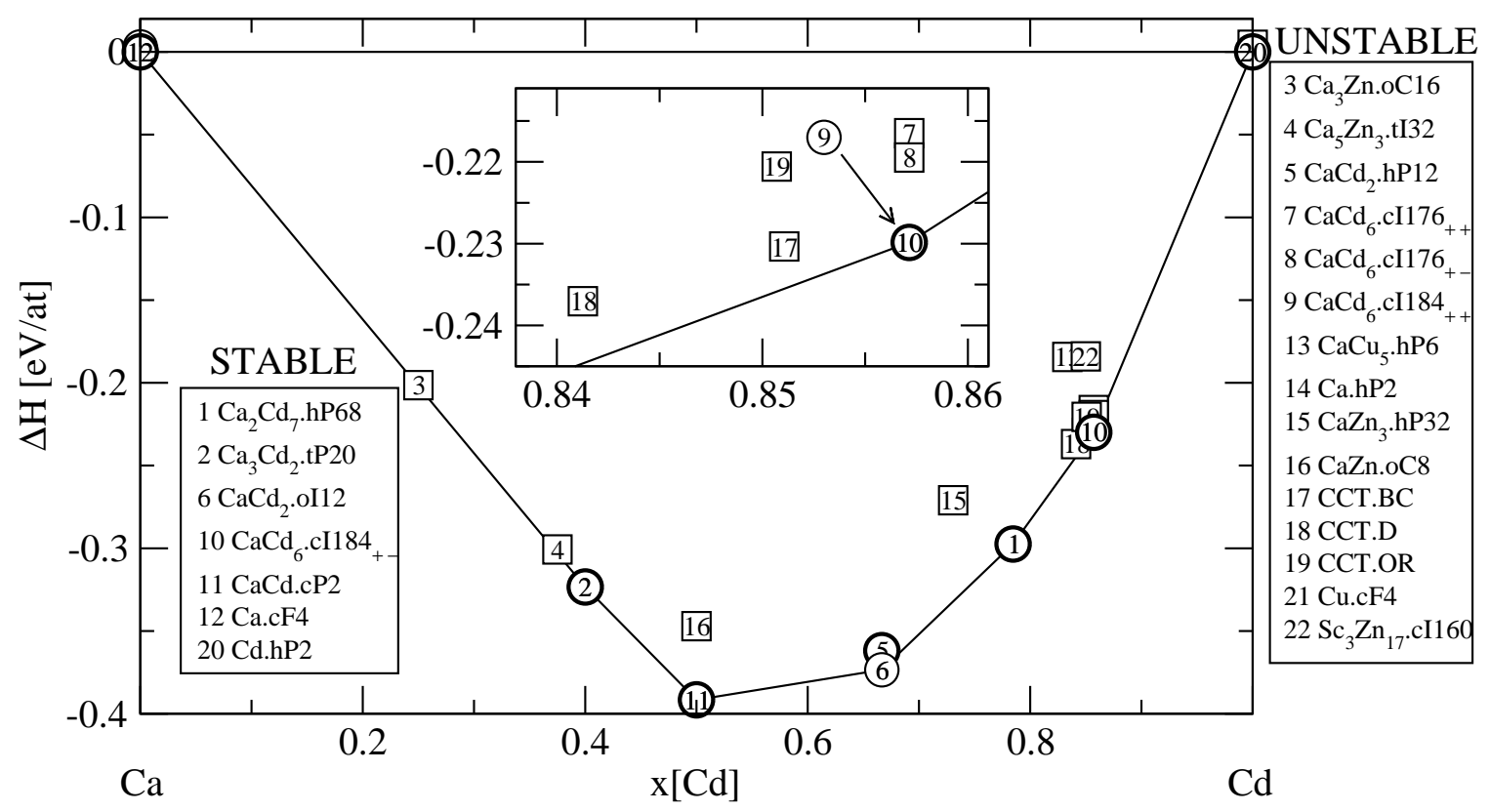

Figure 2: Enthalpies of formation of Ca-Cd compounds. Notation: heavy circles indicate known low temperature phases; light circles indicate known high temperature phases; squares indicate either structures not reported in Ca-Cd system. Line sigments connect vertices of convex hull. Legends list calculated stable structures (left) and unstable structures (right). Inset shows details near quasicrystal-forming composition.

not been well established. Most likely, according to our findings, the presumed low temperature hP12 phase is actually a metastable phase, and the nominal high temperature oI12 phase is actually stable all the way to low temperatures.

The established phase diagram [5] lists a phase $\mathrm{Ca}_{3} \mathrm{Cd}_{17}$, of unknown structure, which is claimed to exist from $\mathrm{T}=0 \mathrm{~K}$ up to melting, at a composition extremely close to $\mathrm{CaCd}_{6}$. Thermodynamic rules governing alloy phase diagrams suggest that either $\mathrm{Cd}_{6} \mathrm{Ca}$ or this unknown phase should actually be stable at high temperature only, because the probability is low that distinct phases coexist over an extended temperature range when they are close in composition.

One candidate for the $\mathrm{Ca}_{3} \mathrm{Cd}_{17}$ phase (based on atomic size ratio and chemical similarity) is $\mathrm{Sc}_{3} \mathrm{Zn}_{17} . c \mathrm{I} 160$. This structure differs from $\mathrm{CaCd}_{6} . \mathrm{cI} 176$ because the innermost $\mathrm{Cd}_{4}$ tetrahedra (see Fig. 1) are missing. We find the energy of this structure is substantially above the convex hull, confirming that occupancy of the inner shell is energetically favorable but leaving open the question if occupancy by four $\mathrm{Cd}$ atoms is the optimum.

Other candidates for the structure of the phase named $\mathrm{Ca}_{3} \mathrm{Cd}_{17}$ are the cluster of additional structures just to the left of $\mathrm{Cd}_{6} \mathrm{Ca}$ in Fig 2. These are postulated icosahedral quasicrystal approximant structures based on canonical cell decorations, as discussed below. The proliferation of many distinct nearly degenerate structures, at energies slightly above the convex hull, is consistent with the proposal of entropic stabilization of quasicrystals. We conclude that the observed phase named $\mathrm{Ca}_{3} \mathrm{Cd}_{17}$ is most likely the icosahedral phase and that it is stable at high temperatures only. 


\section{CANONICAL CELL TILING MODEL}

Our Ca-Cd decoration models utilize tilings of canonical cells [18], whose vertices form maximal-density packings of icosahedral clusters linked along 2-fold and 3-fold icosahedral directions. Space is divided into 4 kinds of "canonical cells", denoted A,B,C and D. Our decoration rule for $\mathrm{A}, \mathrm{B}$ and $\mathrm{C}$ cells was inferred from the refined structure of the $\mathrm{Ca}_{13} \mathrm{Cd}_{76} . \mathrm{cP} 792$ approximant [19], that we identified as the "2/1 ABC" tiling. The resulting A-cell decoration, when applied to the " $1 / 1 \mathrm{~A}$ " tiling, reproduces the known $M \mathrm{Cd}_{6}$ structure. The known quasicrystal approximants contain no D-cells and thus do not imply a specific decoration rule for D-cell interiors. Instead we constructed some plausible variants by hand and selected the lowest energy one. Our decoration rule does not impose specific orientations for the cluster center tetrahedra.

The canonical cell decoration model implies decorations of prolate- and oblate-rhombahedra that are consistent with an earlier proposal [8] for the $1 / 1$ cubic approximant. An advantage of the canonical cell approach is that it can be systematically extended to model higher approximants and also the icosahedral phase.

Several canonical cell approximants were small enough that we could perform total energy calculations (see Fig. 2). In addition to the cubic 1/1 approximant, these were three rhombohedral structures: (i) "BC"-tiling $\left(\mathrm{Ca}_{13} \mathrm{Cd}_{61}\right)$, (ii) "D"-tiling $\left(\mathrm{Ca}_{20} \mathrm{Cd}_{102}\right)$ and (iii) "OR"-tiling $\left(\mathrm{Ca}_{43} \mathrm{Cd}_{245}\right)$, where $\mathrm{Ca}_{X} \mathrm{Cd}_{Y}$ are formulae per primitive cell. The OR tiling contains all four kinds of cells (cell content $\mathrm{A}_{6} \mathrm{~B}_{3} \mathrm{C}_{3} \mathrm{D}$ ), and its primitive cell is a golden oblate rhombohedron with edge length $a \sim \tau^{3} a_{q}$, with "quasilattice constant" (Penrose rhombahedron edge length) $a_{q} \sim 5.7 \AA$.

\section{ORIENTATIONAL CORRELATIONS}

We turn now to the energetics of orientational ordering in the cI176 structure. Consider two realizations of the cI176 structure, identical except for the orientations of the $\mathrm{Cd}_{4}$ tetrahedra at cluster centers. Since the tetrahedron takes two orientations, we can assign an Ising-like spin variable \pm to each one. Assign a tetrahedron the + sign if one of its vertices falls along the direction $(1,1,1)$, and assign it a - sign if instead one of its vertices falls along the direction $(-1,-1,-1)$. If the tetrahedron at the unit cell vertex takes the + orientation and the cell center tetrahedron is also in the + orientation, then the cell vertex and center are equivalent and the symmetry is body centered, hence the Pearson symbol cI176. If, on the other hand, the tetrahedron at the body center takes the - orientation then the centering translational symmetry is broken and the stucture becomes primitive cubic (Pearson symbol cP176) instead of body-centered.

Our calculations show the antiferromagnetic +- configuration is favored. To guage the validity of this result it is important to check convergence in the density of the $k$-point mesh, the cutoff energy and the sensitivity to choice of pseudopotential and the density functional. Table I presents our study. First we vary the $k$-point mesh, from $1 \times 1 \times 1$ (the $\Gamma$ point) up to $4 \times 4 \times 4$ (all Monkhorst-Pack meshes). The table presents the convergence of each structure energy separately as well as the energy difference. All other computational parameters were held fixed during these calculations: medium precision (specifies cutoff energy $168 \mathrm{eV}$ ); ultrasoft pseudopotential; Ceperly-Alder LDA; no atomic relaxation. In the next series we hold constant the k-point density (we use only the $\Gamma$ $k$-point for speed) and test the convergence in precision going from low (cutoff $126 \mathrm{eV}$ ) to medium (cutoff $168 \mathrm{eV}$ ) to high (cutoff $210 \mathrm{eV}$ ). Next, continuing with medium precision and the $\Gamma$ point, we compare unrelaxed energies with partial relaxation (only relaxing clusters (a) and (b) as defined in Fig. 1) and full relaxation in which all atoms can move. 
Table I: Energy convergence studies for CaCd6.cI176 ++ and +- configurations, and their difference $\Delta=E_{++}-E_{+-}$. All units are $\mathrm{eV}$ per simple cubic cell.

\begin{tabular}{l|r|r|r||l|r|r|r}
\hline Setting & $\mathrm{E}_{++}$ & $\mathrm{E}_{+-}$ & $\Delta$ & Setting & $\mathrm{E}_{++}$ & $\mathrm{E}_{+-}$ & $\Delta$ \\
\hline $1 \times 1 \times 1$ & -309.161 & -309.260 & 0.099 & $2 \times 2 \times 2$ & -309.814 & -309.975 & 0.161 \\
$3 \times 3 \times 3$ & -309.664 & -309.754 & 0.090 & $4 \times 4 \times 4$ & -309.653 & -309.780 & 0.127 \\
\hline low & -278.848 & -278.956 & 0.108 & unrelaxed & -309.161 & -309.260 & 0.099 \\
medium & -309.161 & -309.260 & 0.099 & partial & -311.902 & -311.995 & 0.093 \\
high & -309.662 & -309.761 & 0.099 & full & -312.243 & -312.317 & 0.074 \\
\hline
\end{tabular}

Table II: Energies of Ising decorations in double-length structures. Units are eV per doubled cell.

\begin{tabular}{l|r|r|r||l|r|r|r} 
Config & $\mathrm{E}_{0}$ & $\mathrm{dE}$ & $\mathrm{H}$ & Config & $\mathrm{E}_{0}$ & $\mathrm{dE}$ & $\mathrm{H}$ \\
\hline++++ & -617.958 & 0 & $C+16 J_{1}+12 J_{2}$ & +++- & -618.031 & -0.072 & $C+8 J_{2}$ \\
+-+- & -618.178 & -0.219 & $C-16 J_{1}+12 J_{2}$ & ++-- & -617.986 & -0.028 & $C+4 J_{2}$ \\
\hline
\end{tabular}

The maximum displacement is $0.23 \AA$ for partial relaxation and $0.30 \AA$ for full relaxation, always concentrated in the innermost dodecahedral shell. Specifically, those Cd atoms in this shell that adjoin a cluster center vacancy see the largest relaxations.

Given the Ising-like $Z_{2}$ symmetry of the order parameter (one of two orientations) it is appropriate to model the energy using an Ising-model Hamiltonian. Including sufficiently far-neighbor interactions we can surely capture the energetics accurately. However, we have only a single energy difference to work with here, so we can extract only one coupling. Assume this is the nearest-neighbor coupling, along the cube body diagonal, and call it $J_{1}$.

Each BCC lattice site has 8 nearest neighbors, each of which reverses sign when going from ++ to +- . There are two lattice sites per simple cubic cell, but we must avoid overcounting the bonds, since each bond is shared by two lattice sites. Hence we conclude that $\Delta=16 J_{1}$, or $J_{1} \approx+0.004 \mathrm{eV}$ (using the fully relaxed value). Because the value of $J_{1}$ is positive the interaction is antiferromagnetic.

To determine the transition temperature for this system we wrote a simple Monte Carlo program to simulate the BCC Ising antiferromagnet. Actually, by simply reversing the sign convention for spins at body-center sites, the BCC antiferromagnet can be seen to be equivalent to the BCC ferromagnet. According to our simulations, the transition temperature should be around $\mathrm{T}=350 \mathrm{~K}$.

Because this temperature is well above the reported transition temperature of $\mathrm{T}=100 \mathrm{~K}$, we investigated the role of further neighbor interactions. If further-neighbor couplings have appropriate signs the magnetism can become frustrated, lowering the transition temperature and leading to spatial modulation of the low temperature structure.

The next nearest neighbor lies along the cube edge, and we will call this coupling $J_{2}$. In order to extract values of $J_{2}$ we need to study a larger cell, so we doubled the cell along the x-axis, and considered the configurations denoted $\sigma_{1}^{v} \sigma_{1}^{c} \sigma_{2}^{v} \sigma_{2}^{c}$ in which the cube vertex of the first cell has spin $\sigma_{1}^{v}$, etc. Owing to the large number of atoms present we report here only the results of $\Gamma$ point calculations.

Fitting these energies to a two-coupling Ising model yields values of $J_{1}=+0.0068$ and $J_{2}=-0.0102 \mathrm{eV}$. Because $J_{2}$ is negative, the next-nearest interaction proves ferromagnetic, which will increase the transition temperature and also will not lead to superlattice ordering. In other words, the Ising model presented so far is rather inconsistent with the experimental findings. 


\section{DISCUSSION}

Given that our results disagree with experiment in both superlattice ordering and transition temperature it is clear that additional study is needed, especially the study of additional tetrahedron orientations. We restricted our attention to tetrahedron orientations belonging to subsets of a basic cube (based on the cI176 structure), in order to have a simple Ising-model description. There are many more possible orientations. Among these are the cuboctahedron of the cI184 structure, which our calculations (see Fig. 2) already show is prefered over cI176. A recent study of the $M \mathrm{Cd}_{6}$ structure family by Gomez and Lidin [11] finds additional partially occupied sites, proposing the new Pearson type $\mathrm{CaCd}_{6} . \mathrm{cI} 232$. If an order-disorder transition exists in this new structure, it will be at a lower temperature than found in our initial study because: (1) the energy differences among orientations are generally lower; (2) the order parameter has a higher symmetry than the Ising spins so there is a higher orientational entropy.

\section{ACKNOWLEDGEMENTS}

We wish to acknowledge useful discussions with R. Tamura and C.L. Henley. This work was supported in part by NSF grant DMR-0111198

\section{REFERENCES}

1. A. P. Tsai, J. Q. Guo et al., Nature, 408, 537, (2000).

2. J. Q. Guo and E. Abe and A. P. Tsai, Phys. Rev. B, 62, R14605, (2000).

3. J. Z. Jiang and C. H. Jensen and A. R. Rasmussen and L. Gerward, Appl. Phys. Lett., 78, 1856, (2001).

4. P. Villars Pearson's Handbook, Desk Edition (ASM Int., Materials Park, Ohio, 1997).

5. T. B. Massalski et al. eds. Binary Alloy Phase Diagrams (ASM Int., Materials Park, Ohio, 1990).

6. A. Palenzona, J. Less-Common Metals, 25, 367, (1971).

7. G. Bruzzone, G. Chim. Ital., 102, 234, (1972).

8. H. Takakura, J. Guo and A. P. Tsai, Phil. Mag. Lett., 81, 411, (2001).

9. Y. Ishii and T. Fujiwara, Phys. Rev. Lett., 87, 206408, (2001).

10. R. Tamura, K. Edagawa et al., J. Non-cryst. Solids (to appear, 2004).

11. C. P. Gomez and S. Lidin, Phys. Rev. B, 68, 024203, (2003).

12. R. Tamura, Y. Murao, et al., Jpn. J. Appl. Phys., 41, L524, (2002).

13. R. Tamura, K. Edagawa, et al., Phys. Rev. B, 63, 132204 (2003).

14. G. Kresse and J. Hafner, Phys. Rev. B, 47, RC558, (1993).

15. G. Kresse and J. Furthmuller, Phys. Rev. B, 54, 11169, (1996).

16. G. Kresse and J. Hafner, J. Phys. Condens. Matter 6, 8245 (1994)

17. G. Kresse and J. Joubert, Phys. Rev. B, 59, 1758, (1999).

18. C. L. Henley, Phys. Rev. B, 43, 993, (1991).

19. P. C. Gomez and S. Liden, preprint (2002). 